\title{
Increase in Cancer Patient Load during COVID-19 Pandemic: The Faridabad Experience
}

\section{Sumant Gupta ${ }^{1}$ Anamika Pandey ${ }^{2} \quad$ Neha Gupta $^{1} \quad$ Faran Naim ${ }^{1} \quad$ Rakesh Gupta $^{3} \quad$ Bhaskar Das ${ }^{4}$}

\author{
${ }^{1}$ Department of Medical Oncology, Sarvodaya Hospital and \\ Research Centre, Faridabad, Haryana, India \\ 2Department of Psycho-Oncology, Sarvodaya Hospital and Research \\ Centre, Faridabad, Haryana, India \\ ${ }^{3}$ Department of Internal Medicine, Sarvodaya Hospital and Research \\ Centre, Faridabad, Haryana, India \\ ${ }^{4}$ Department of Microbiology, Sarvodaya Hospital and Research \\ Centre, Faridabad, Haryana, India
}

Address for correspondence Sumant Gupta, MD, DM, Department of Medical Oncology, Sarvodaya Hospital \& Research Center, Sector 7, Faridabad 121006, Haryana, India

(e-mail: drsumantgupta@gmail.com).

\section{South Asian J Cancer 2021;10:36-38.}

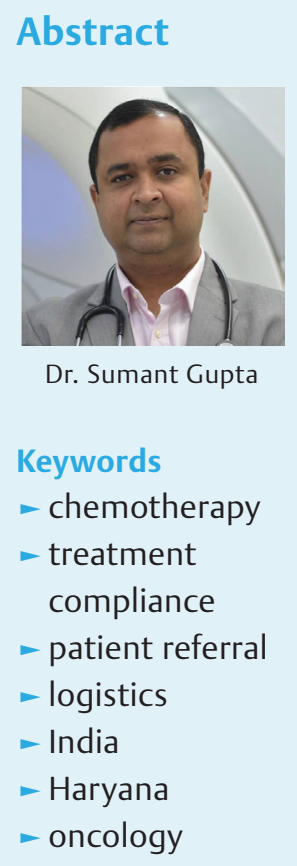

Introduction Coronavirus disease 2019 (COVID-19) has affected oncology care differently across the world. We evaluated our experience of infusional chemotherapy during the active phase of ongoing pandemic.

Methods Prospectively collected month wise data from January 2019 to November 2020 was compared between the 2 years.

Results A total of 6,003 chemotherapy infusions were administered between January 1 , 2019 and November 30, 2020 (2,548 in 11 months of 2019 and 3,455 in the same 11 months of 2020). Between May 1 and October 31, 2020, 2,337 chemotherapy infusions were administered to 570 patients all of whom were also tested for COVID-19 positivity, of which 65 (11.4\%) were COVID-19 positive. The majority (63/65; 97\%) could receive their chemotherapy infusions safely.

Discussion Paradoxically, our hospital recorded an increase in the number of cancer patients receiving infusional chemotherapy in 2020, with a linear increase in the cancer case being treated (from 309 in June to 398 in November 2020). We believe that this was possible because cancer patients wanted treatment near their homes to avoid/minimize risk of exposure to COVID-19, cross state border travel restrictions was an additional roadblock, and our quality of service provided earned the trust of cancer patients.

\section{Introduction}

The coronavirus disease 2019 (COVID-19) pandemic has usually resulted in a focus on this infection at the cost of non-COVID patient management. ${ }^{1}$ Our center paradoxically witnessed an increase in cancer patients administered infusional chemotherapy during the active phase of the ongoing pandemic. We present these interesting results and discuss the possible reasons for the same.

DOI https://doi.org/10.1055/s-0041-1726159 ISSN 2278-330X.

How to cite this article: Gupta S, Pandey A, Gupta N, Naim F, et al. Increase in Cancer Patient Load during COVID-19 Pandemic: The Faridabad Experience South Asian J Cancer 2021;10(1):36-38.

\section{Methods}

This is a retrospective analysis of prospectively collected data of all cancer patients treated at our center with infusional chemotherapy between January and November 2020. This was then compared to the equivalent data for the corresponding months in the previous year (2019). We also evaluated the COVID-19 status of cancer patients between May 1, 2020 and October 31, 2020. COVID-19

(C) 2021. MedIntel Services Pvt Ltd.

This is an open access article published by Thieme under the terms of the Creative Commons Attribution-NonDerivative-NonCommercial-License, permitting copying and reproduction so long as the original work is given appropriate credit. Contents may not be used for commercial purposes, or adapted, remixed, transformed or built upon. (https://creativecommons.org/licenses/by-nc-nd/4.0/).

Thieme Medical and Scientific Publishers Private Ltd A-12, Second Floor, Sector -2, NOIDA -201301, India 
positive patients were also followed up while under our care to document their immediate outcome.

\section{Results}

A total of 6,003 chemotherapy infusions were administered to cancer patients at our center between January 1, 2019 and November 30, 2020 (2,548 in 11 months of 2019 and 3,455 in the same 11 months of 2020). Their month wise distribution is compared in -Fig. 1. -Fig. 2 shows the percentage change between the corresponding months of the 2 years. The month of August was the only month with a negative growth for 2020 as compared to 2019.

Between May 1 and October 31, 2020, 2,337 chemotherapy infusions were administered to 570 patients all of whom were also tested for COVID-19 positivity. A total of 65/570 (11.4\%; 39 males and 26 females; aged $13-80$ years) were detected to have COVID-19 infection. Their underlying malignancies were hematological (18/65; 28\%), lung (10/65; 15\%), breast $(9 / 65,13 \%)$, head and neck $(8 / 65,12 \%)$, gynecological ( $7 / 65 ; 10 \%)$, genitourinary $(4 / 65 ; 6 \%)$, and others $(9 / 65 ; 17 \%)$. The majority $(63 / 65 ; 97 \%)$ could receive their chemotherapy infusions safely. Two patients (both with acute myeloid leukemia) succumbed to COVID-19 (one before start of anticancer therapy and the other while on day 7 of the induction treatment).

\section{Discussion}

The COVID-19 pandemic has had a more profound effect on some parts of the world including India and United States. ${ }^{1}$ It hit the Southeast Asian nations harder than the rest of the world. On March 24, 2020, our central government imposed a nationwide lockdown in an effort to protect our population. ${ }^{2}$ All emergency services, including healthcare, were permitted to continue operations. However, management of non-COVID-19 illnesses was shunted to the background. The fear of a potentially life-threatening infection invisibly lurking round the corner was coupled with limited knowledge about the virus and triggered diverse precautionary measures-both in the community and healthcare facilities. ${ }^{3}$ Most nonemergency surgical procedures, routinely scheduled hospital visits as well as clinical trial were postponed, suspended,

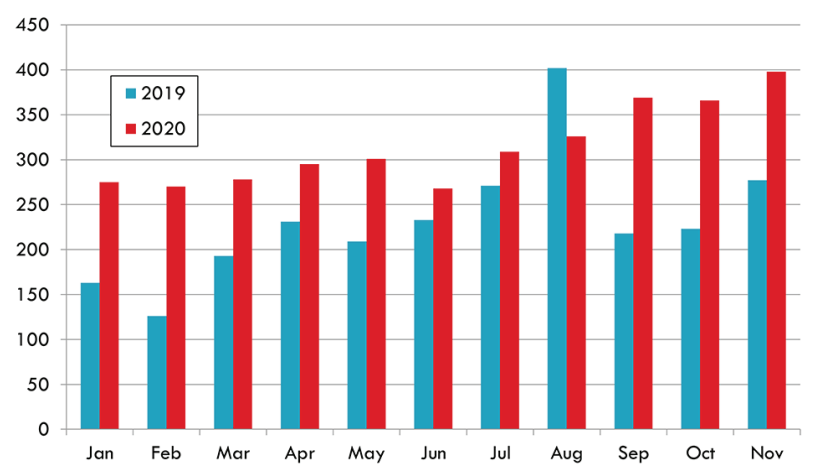

Fig. 1 Chemotherapy administrations.

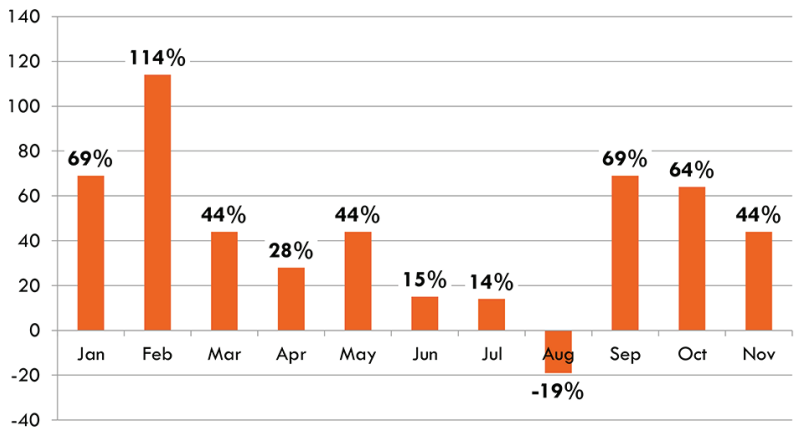

Fig. 2 Chemotherapy administrations-difference between 2020 and 2019 (percentage change).

or completed with great difficulty. ${ }^{4}$ News from the western world (United States, France, Italy, Spain) confirmed healthcare facilities being overwhelmed, COVID-19 patients succumbing with lung-related features and involvement of children (a group that had been considered as relatively safe from COVID-19). 5,6

Cancer patients were particularly vulnerable because they felt trapped between the devil and the deep sea. While venturing out meant taking the risk of getting infected with COVID-19, staying at home meant that the cancer would continue to grow, metastasize, and could become incurable. Conflicting guidelines put forth by various authorities and oncological bodies added to the confusion. ${ }^{7.8}$ Surgical procedures were the ones affected the most, with patients being referred for radiation and/or systemic therapy (chemotherapy, targeted therapy) as a stop-gap arrangement to tide over the crisis.

Fortunately, the severity of the infection and its mortality were significantly lower in India as compared to many other countries. ${ }^{9}$ This was due to a combination of some protective immunity among Indian as well as innovative treatment implementations ${ }^{10,11}$

Paradoxically, Sarvodaya Hospital, Faridabad, had a unique experience. As compared to 2019, we recorded an increase in the number of cancer patients receiving infusional chemotherapy in 2020. This was consistent across all the months, except for August. In fact, if we focus solely on the numbers for 2020, it will be clear that infusional chemotherapies administered remained essentially consistent from Jan to May (before the pandemic hit Haryana-168 daily new cases in the entire state on May 31, 2020; - Fig. 3)..$^{12}$ In the month of June new cases of COVID-19 peaked at 534 daily in Haryana, almost three times that in the previous month. This is also the month when our hospital saw the least number of cases receiving infusional chemotherapy. Thereafter, there was a linear increase in the cancer case being treated (increased from 309 in June to 398 in November 2020). From - Figs. 1 and 2, it becomes evident that the percentage 'reduction' in cases in August 2020 as compared to August 2019 is actually due to an unusually high number of patients that we treated in August 2019 (404) that we cannot explain. An alternate explanation for dip in August 2020 could be the steep rise in the number of new COVID-19 cases in the state (from 793 on August 1 to 


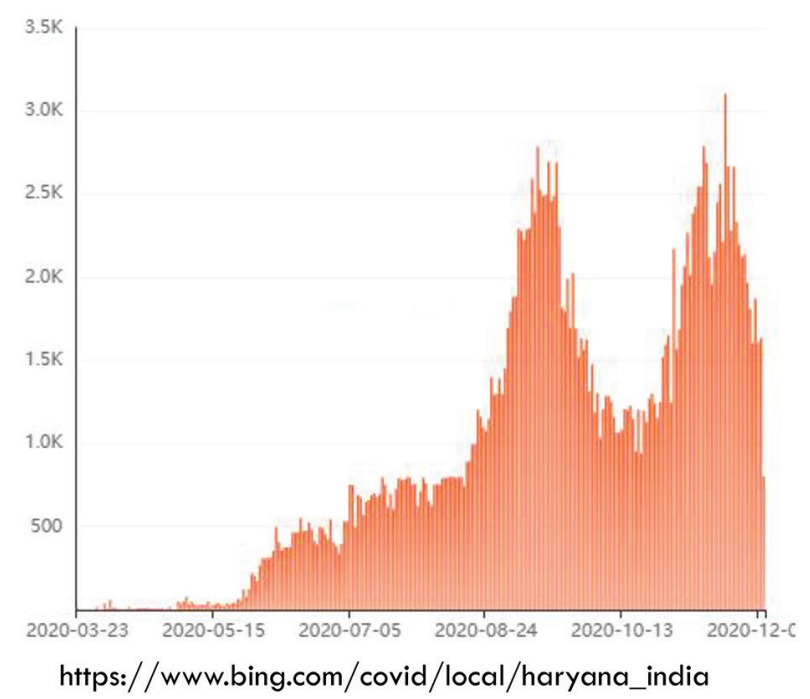

Fig. 3 Daily new coronavirus disease 2019 (COVID-19) cases in the state of Haryana, India.

1,450 on August 31,2020 ) that led to patients on treatment fearing to leave their homes. The third reason could be the unlocking of the Delhi/NCR borders in July that would have allowed patients to revert to their original oncologists (not needing to continue their treatment at our hospital).

We believe that the reason why we consistently saw a linear increase in cancer chemotherapy cases at our hospital from June to November 2020 is as follows:

1. Cancer patients wanted treatment near their homes to avoid/minimize risk of exposure to COVID-19.

2. Cross border travel restrictions resulted in patients no longer having the ease of seeking treatment in NCR.

3. The quality of service provided by Sarvodaya Hospital was sufficient to earn the trust of cancer patients who experienced them.

Our unique selling price is that we rose to the challenge, modified hospital standard operating procedures (SOPs), and continued to provide medical oncology services while simultaneously tackling the COVID-19 pandemic. In the 6-month period beginning May 1, 2020, our department registered 570 new patients with cancer who were administered 2,337 chemotherapy infusions.

\section{Conclusion}

In 2020, our center experienced increase in cancer patients requiring infusional chemotherapy during the COVID-19 pandemic in compared to 2019. This was likely due to the inability of local patients to travel to the NCR due to lockdown restrictions imposed to prevent further spread of COVID-19 in the community. We were able to gear up with new SOPs to continue providing standard medical oncology services to our cancer patients. We implemented this without any increased mortality. Till the time as herd immunity or vaccination brings the pandemic under control, we will follow regular COVID-19 testing and precautions while optimizing cancer management on the basis of better understanding of both the diseases.

Conflict of Interest

None.

\section{References}

1 Parikh PM, Bapna A, Krishna MV, Mehta P, Aggarwal S, Gulia A. COVID-19 testing in India in comparison to the rest of the world. If Indian testing strategy was replicated in the other top 15 COVID-19 affected countries in the world, the status would be startlingly different. Indian J Med Sci 2020;72:107-109

2 https://en.wikipedia.org/wiki/COVID-19_pandemic_lockdown_in_India. Accessed February 10, 2021.

3 Parikh P, Mehta P, Bansal S, et al. Protecting health-care professionals and workers (other than COVID-19 management facilities) from contamination during COVID-19 pandemic. Int J Med Sci 2020;72:3-4

4 Parikh PM, Mehta P, Krishna Kumar MVT, Govind Babu K. Clinical trials facing "Serious Adverse Events" during the ongoing COVID-19 pandemic. Indian J Med Paediatr Oncol 2020;41:295-298

5 Di Stadio A, Ricci G, Greco A, de Vincentiis M, Ralli M. Mortality rate and gender differences in COVID-19 patients dying in Italy: a comparison with other countries. Eur Rev Med Pharmacol Sci 2020;24(8):4066-4067

6 Parikh PM, Mehta P, Aggarwal S. Important COVID-19 update new life-threatening syndrome in pediatric patients? Intl J Mol Immunol Oncol 2020;5:42-43

7 Tartarone A, Lerose R. COVID-19 and cancer care: what do international guidelines say? Med Oncol 2020;37(9):80 10.1007/ s12032-020-01406-5

8 Gundavda MK, Gundavda KK. Cancer or COVID-19? A review of guidelines for safe cancer care in the wake of the pandemic. SN Compr Clin Med 2020:1-11 doi: 10.1007/s42399-020-00632-2

9 Mehta P, Parikh P, Aggarwal S, et al. Has India met this enemy before? From an eternal optimist's perspective: SARS-CoV-2. Indian J Med Sci 2020;72(1):8-12

10 Gundavda KK, Parikh PM. Convalescent plasma therapy for COVID-19 still has the potential to save lives - the ICMR PLACID study dissected. Int J Mol Immuno Oncol 2021;6(1):47-49. doi: 10.25259/IJMIO_31_2020

11 Budhiraja S, Dewan A, Aggarwal R, et al. Effectiveness of convalescent plasma therapy in Indian patients with COVID-19. Lancet 2020. Accepted for publication Available at https:// papers.ssrn.com/sol3/papers.cfm?abstract_id=3726179

12 https://www.bing.com/covid/local/haryana_india. Accessed February 10, 2021. 